\title{
Article
}

\section{A Simple Method for Acquisition and Inoculation of Brassica Yellows Virus from Transgenic Plants and Frozen Infected Leaves by Aphids}

\author{
Deng-Pan Zuo ${ }^{1}$, Xiang-Ru Chen ${ }^{1}$, Ru-Jian Hu ${ }^{1}$, Tian-Yu Zhao ${ }^{1}$, Xiao-Yan Zhang ${ }^{1}$, Yan-Mei Peng', \\ Ying Wang ${ }^{1}$, Da-Wei $\mathrm{Li}^{2}$, Jia-Lin Yu${ }^{2}$ and Cheng-Gui Han ${ }^{1, *}$ \\ 1 State Key Laboratory for Agrobiotechnology and Ministry of Agriculture Key Laboratory of Pest \\ Monitoring and Green Management, College of Plant Protection, China Agricultural University, Beijing \\ 100193, China; B20173190806@cau.edu.cn (Z.DP.); chxr8628@163.com (C.XR.); hurujian@cau.edu.cn (H.RJ.); \\ ty_zhao@163.com (Z.TY.); xiaoyan433@163.com (Z.XY.); pengyanmei7628@163.com(P.YM.) ; \\ yingwang@cau.edu.cn (W.Y.) \\ 2 State Key Laboratory of Agro-Biotechnology and Ministry of Agriculture Key Laboratory of Soil \\ Microbiology, College of Biological Sciences, China Agricultural University, Beijing, 100193, \\ Dawei.Li@cau.edu.cn (L.DW.); yjl@cau.edu.cn (Y.JL.) \\ * Correspondence: hanchenggui@cau.edu.cn (H.CG.); Tel.: +86-10-6273-2619
}

\begin{abstract}
Brassica yellows virus (BrYV) is a tentative species of the genus Polerovirus, which occurs widely and mostly damage Brassicaceae plants in East Asia. Since BrYV could not be transmitted mechanically, an insect transmission method is required for further virus research. Here, a reliable and unrestricted method was described, in which non-viruliferous aphids (Myzus persicae) acquired BrYV from transgenic Arabidopsis thaliana plants with virus full length genome germinated from seeds and frozen infected leaves were used to transmit the virus to healthy plants, and there was no significant difference in acquisition rate though transmission rate from frozen infected leaves was somehow lower compared to fresh infected leaves. This novel simple method could be applied to preservation of virus inocula, evaluation of variety resistance to BrYV, biological research on interaction among BrYV, aphid and host, which also provide a new idea on establishing a basic method using virus genomic transgenic plants or frozen infected leaves for other poleroviruses research.
\end{abstract}

Keywords: Brassica yellows virus (BrYV); Myzus persicae; transgenic plants with BrYV full length genome; frozen BrYV infected plants; acquisition and transmission

\section{Introduction}

Viruses in the Polerovirus (family Luteoviridae) cause plant diseases that are notoriously difficult to manage [1]. Poleroviruses are positive single-stranded RNA (+ssRNA) viruses. The virions are spherical, about $25-35 \mathrm{~nm}$ in diameter, and non-enveloped. The virions are composed of coat protein and genomic RNA (gRNA) [2]. Polerovirus are widely distributed worldwide and can infect a variety of plants such as Cruciferae, Polygonaceae, Cucurbitaceae, Gramineae plants causing serious diseases and economic losses [3-5]. Polerovirus are phloem restricted and cannot be transmissible mechanically in natural condition. Naturally, it can only be transmitted by aphids in a circulative, non-propagative manner meanwhile it can also be spread by agroinfiltration or grafting under artificial conditions $[6,7]$.

Brassica yellows virus (BrYV) is a tentative newly identified species in the genus of Polerovirus, which is closely related to TuYV (Turnip yellows virus) $[8,9]$. BrYV are widely spread in China as well as in South Korea and Japan $[4,10,11]$. BrYV has a wide range of hosts, which mainly infects the Cabbage (Brassica oleracea var.capitata), Chinese cabbage (B.pekinensis), Cauliflower (B.oleracea var.botrytis), Mustard (B.juncea), Turnip (Raphanus sativus var.oleifera) and other Brassicaceae crops, usually causing leaf malformation and yellowing in the field [4,9-11]. BrYV has at least three 
genotypes (BrYV-A, B, and C) according to sequence analysis, the full-length infectious cDNA clones of these three genotypes have been developed successfully [8,10-13]. Previously work have shown that the full-length amplicon of the virus has been successfully transferred into Arabidopsis thaliana and obtained 2 stable genetically transgenic lines named 111 and 412 which all exhibited severe symptoms including dwarfing and the purple leaves [14]. BrYV P0 protein interacted with the plant SKP1 contributes to its stability thereby evading autophagy and proteasomal degradation [15]. In addition, Brassica yellows virus P0 protein could impair the antiviral activity of NbRAF2 in Nicotiana benthamiana by altering its localization pattern to facilitate virus infection [16].

For poleroviruses are phloem limited and the infected plants often have less virus titre, the infected plants could not maintain for a long time, therefore the storage and utilization of the virus inocula source are very important [6]. Moreover, the infected plants collected from the field usually are not infected by a single virus which greatly limits the simplicity and reliability of the research on poleroviruses. Boissinot et al (2017) reported that extracting the virus-infected plants to obtain virus virions and use the Agrobacterium tumefaciens containing the virus infectious clone to infect plants for feeding non-viruliferous aphids for inoculation [17]. The full-length genomic RNA of PLRV (Potato leafroll virus) were transferred into potato and tobacco and the transgenic plants virus titre was higher than the infected wildtype plants. Moreover, the virus in the transgenic plants could spread to the epidermal cells and was transmitted more efficiency through aphid feeding [18,19]. Shikata et al (1977) reported that the RBSDV (Rice black-streaked dwarf virus) were investigated by injection into SBPH (Small brown planthopper) artificially from RBSDV infected rice leaves could transmit the virus to healthy plants [20]. Moreover, SBPH could directly acquire RBSDV from frozen infected rice leaves and transmitted to a susceptible rice variety [21]. Zhang et al (2007) provide a simple, rapid and reliable method as described by which virus-free SBPH acquired RSV (Rice stripe virus) from frozen infected rice leaves and transmitted the virus to healthy rice plants [22]. These reports provided valid methods to us to solve the problem of insect vector acquiring virus from frozen inocula and virus infected plants to establish a basic research method in breeding and genetic research on plant disease resistance. Up to now, there are no reference that aphids acquire BrYV from transgenic plants with virus full length genome-germinated from seeds kept in storage and frozen infected leaves as a virus inoculation material. As BrYV infectious cDNA clone could infected model plants like Arabidopsis thaliana and Nicotiana benthamiana mediated by Agrobacterium infiltration [13,14]. For the reason of Agrobacterium infiltration method cannot completely replace natural virus inoculation conditions. Therefore, we develop a simple and reliable method for acquisition and inoculation of BrYV from transgenic plants and frozen infected plants by aphids, which will provide a basic research method for BrYV inocula preserve and transmission and make it better to unravel the interactions among aphid, virus and host. In addition, this method also provided a new idea on establishing a similar transmission system for other poleroviruses research.

\section{Materials and Methods}

\subsection{Virus resource}

Transgenic A. thaliana line 412 with full-length cDNA amplicon of BrYV-C that constitutively expressed viral genomic RNA of BrYV [11,13], and the seeds used were the seventh generation. $A$. thaliana line 412 seedlings at 3-4 weeks old grown in a greenhouse or a chamber could be used as virus inocula. Alternatively, 4- to 5-week-old A. thaliana plants infiltrated with Agrobacterium tumefaciens strain $\mathrm{C} 58 \mathrm{CI}$ containing BrYV full-length cDNA clone were also used as virus inocula. All the plants were grown at $24{ }^{\circ} \mathrm{C} / 10$-h light and $22^{\circ} \mathrm{C} / 8$-h dark cycle. 


\subsection{Identification of aphid species}

In October 2017, aphids were collected from west campus, China Agricultural University, and maintained on a turnip variety Yamei No.1. For aphid species identification, we used PCR to amplified the aphid cytochrome oxidase gene (COI) of the aphids. Firstly, A single aphid total DNA was extracted by CTAB DNA extraction buffer. Added $300 \mu \mathrm{L}$ of CTAB DNA extraction buffer to the tube and mixed well by shaking. Placed the tube on a $65^{\circ} \mathrm{C}$ heater for $30 \mathrm{~min}$ and mixed it well upside down several times. Then added an equal volume of tris-phenolic mixture, centrifuged at 12,000 rpm for $15 \mathrm{~min}$ and aspirated the supernatant for $300 \mu \mathrm{L}$. Added an equal volume of chloroform isoamyl alcohol (24:1) then centrifuged at 12,000 rpm for $15 \mathrm{~min}$, pipetted the supernatant for $200 \mathrm{ul}$ and added an equal volume of isopropanol at room temperature for $10 \mathrm{~min}$. After centrifuging, added $600 \mu \mathrm{L}$ $75 \%$ and $100 \%$ ethanol for washing respectively. Finally, dried the DNA in room temperature then added $30 \mu \mathrm{L} \quad \mathrm{ddH}_{2} \mathrm{O}$ to dissolved. The mitochondrial universal primer (LEP-F: ATTCAACCAATCATAAAGATATTG; LEP-R: AAACTTCTGGATGTCCAAAAAATCA) were used here to amplified the aphid cytochrome oxidase gene (COI) by PCR. Recovered target PCR products from agarose gel electrophoresis of samples by the Gel Extraction Kit (OMEGA) and sent the samples for sequencing, and then blasted the sequence in NCBI (https://www.ncbi.nlm.nih.gov/) for sequence analyzing.

\subsection{Generation of non-viruliferous aphid monoclonal population}

Screening of the aphid colony for separating them to obtain virus-free and monoclonal aphids as described $[23,24]$. Here, we selected one single aphid from the aphid group has been identified species of Myzus persicae for oviposit and reared in healthy $A$. thaliana. Then transferred one nymph to healthy $A$. thaliana and for about 5-10 generation, aphids were randomly selected and analyzed by RT-PCR for confirming BrYV negatively. After that, one non-viruliferous aphid was reared in healthy turnip seedlings (3-4 leaves) in the light incubator at $24{ }^{\circ} \mathrm{C}$ with a $16-\mathrm{h} \mathrm{light} / 8-\mathrm{h}$ dark cycle. For about 10 generation offspring, these non-viruliferous aphids were classified as a population named Mp4331. The selected aphid offspring from single aphid were further confirmed to be negative for BrYV by RT-PCR. Total RNA was extracted from the aphid samples using TRIzol reagent according to the manufacturer's instructions (Invitrogen). Firstly, putted a single aphid into a $2.0 \mathrm{~mL}$ Eppendorf tube and added the steel ball then fitted into the liquid nitrogen, grinded it and immediately centrifuge the sample to $12,000 \mathrm{rpm}$ in a pre-cooled centrifuge. After 10 seconds short centrifugation, $300 \mu \mathrm{L}$ TRIzol was added into the sample and placed horizontally on the experiment table for $5 \mathrm{~min}$ to allowed the sample to be fully lysed. Then added $200 \mu \mathrm{L}$ of chloroform and shaken it for 30 seconds, stewing at room temperature $\left(25^{\circ} \mathrm{C}\right)$ for $5 \mathrm{~min}, 12,000 \mathrm{rpm}$ centrifuged at $4^{\circ} \mathrm{C}$ for $10 \mathrm{~min}$. After that, took the supernatant in a new RNase-free tube and added $300 \mu \mathrm{L}$ of isopropanol at room temperature for $10 \mathrm{~min}$ and then $12,000 \mathrm{rpm}$ for centrifugation for $15 \mathrm{~min}$ at $4{ }^{\circ} \mathrm{C}$. Discarded the supernatant and added $1 \mathrm{~mL}$ of $75 \%$ alcohol to lightly washed the RNA pellet centrifuging at 12,000 rpm for $5 \mathrm{~min}$. Finally, removed the supernatant and dyed at room temperature for 5-10 min, dissolved in $20 \mu \mathrm{L}$ of DEPC pretreated water. The reverse transcription system is carried out according to the instructions of M-MLV reverse transcriptase (Promega). The reverse transcription system was carried out according to the instructions of M-MLV reverse transcriptase (Promega). PCR detection with specific primers (BrY4964F: CATAAAGCCCTTGTCGGCGAG, BrY5635R: GTAGAACACTCGTTGCCTATCC) for the transcriptional products to be detected.

\subsection{Virus acquisition experiments}

The non-viruliferous aphid group (Mp433-1) was confirmed by RT-PCR before feeding. The BrYV transgenic $A$. thaliana grown to 3-4 weeks were used and detached leaves were placed in plantlet bottle for aphids feeding. Frozen infected leaves (The frozen time settings were 180-days and 270-days, respectively) were thawed in Petri dishes containing wet filter paper that retained moisture for at least $4 \mathrm{~h}$ and then transferred to a plantlet bottle at light incubator (Figure 1). Sixty nonviruliferous aphids (2nd-3rd instar) were placed into plantlet bottle. Blank controls were fresh healthy 
plant leaves. After a 48-h acquisition feeding period, the surviving aphids were calculated and transferred from the inocula leaves to healthy A. thaliana seedlings with a brush. Each experiment was repeated at least 3 times.

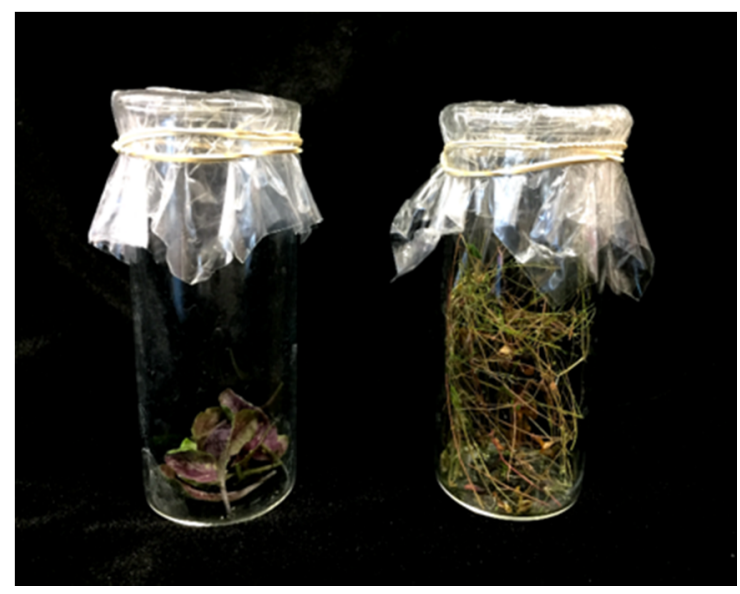

Figure 1. A feeding plantlet bottle, containing Myzus persicae and fresh and frozen $A$. thaliana leaves; The left plantlet bottle with fresh BrYV transgenic $A$. thaliana leaves; The right plantlet bottle with frozen BrYV transgenic $A$. thaliana leaves.

\subsection{Virus transmission experiments}

For aphids after feeding on the line 412, frozen infected leaves and fresh infected A. thaliana leaves were inoculated for virus transmission and detection. Aphid was caged individually when put on the plants and after inoculation which were all removed out for virus detection. For the best transmission efficiency of BrYV which healthy $A$. thaliana plants were inoculated by 1, 2, 4, 6 and 10 aphids (2nd-3rd instar) respectively. After inoculation, aphids were eliminated with insecticide. Each seedling was inoculated individually for 2 days inoculation time. After symptom investigation, the inoculated plants were detected for virus by RT-PCR and western blotting [Figures $2 a, b$ and 3 ]. The aphids fed on healthy leaves were used as negative controls, and each treatment was repeated 3 times. The inoculated plants were grown in a greenhouse at $24^{\circ} \mathrm{C}$ with a $16-\mathrm{h}$ light/8-h dark cycle and were observed for 1-2 weeks.

a

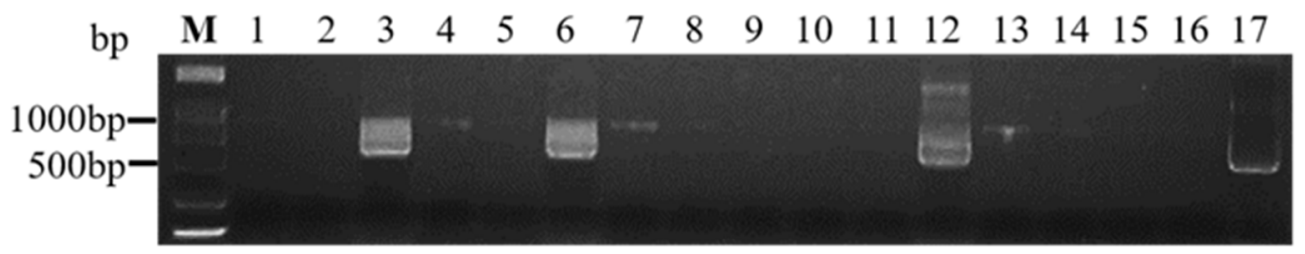

b

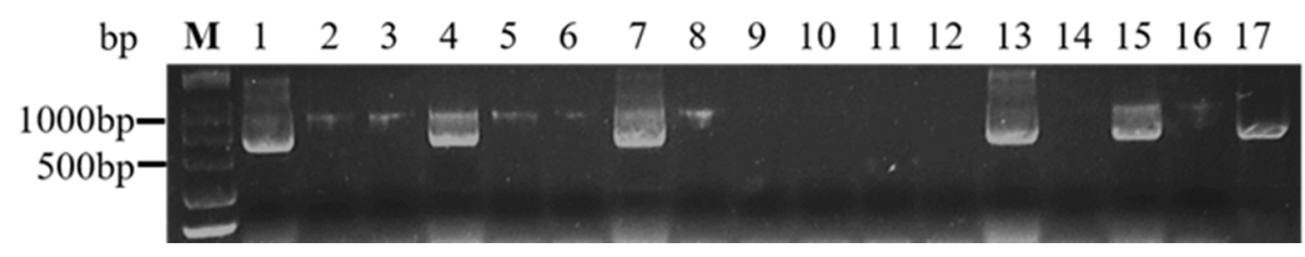

Figure 2. Agarose gel analysis of RT-PCR products from BrYV in A. thaliana plants inoculated by aphid. (a) Aphids acquire the virus from the frozen infected leaves for transmission; (b) Aphids acquire the virus from transgenic A. thaliana line 412 leaves for transmission. M: Marker (DCL 2000, Tsingke); line 1-16: aphids inoculated $A$. thaliana; line 17: positive control. 


\subsection{Detection of $B r Y V$ in aphids and A. thaliana plants}

After removal of the aphids, RT-PCR and western blotting were carried out for virus detection in aphids and $A$. thaliana plants. For aphid detection which were feed on line 412, frozen infected leaves, healthy and fresh infected $A$. thaliana plants, virus detection was described as 2.3. For virus detection of $A$. thaliana, the total RNA was extracted from plants samples using the TRIzol reagent according to the manufacturer's protocol. First-strand cDNA was synthesized using $2 \mu \mathrm{g}$ of treated RNA, oligo d(T) primer, or gene-specific primer and M-MLV Reverse Transcriptase as instructed by the protocol. Primers were designed as described above. For western blotting assay, total proteins were extracted from systemic leaves of aphids inoculated $A$. thaliana using $2 \mathrm{x}$ sodium dodecyl sulfate (SDS) sample buffer $\{100 \mathrm{Mm}$ Tris ( $\mathrm{pH} 6.8), 4 \%(\mathrm{w} / \mathrm{v})$ SDS, $20 \%(\mathrm{v} / \mathrm{v})$ glycerol, and $0.2 \%(\mathrm{w} / \mathrm{v})$ bromophenol blue with subsequent heating at $100^{\circ} \mathrm{C}$ for $10 \mathrm{~min}$ [14-16]. $12.5 \%$ SDS polyacrylamide gel for protein separated by electrophoresis and then transferred to nitrocellulose membrane (GE Healthcare, Buckinghamshire, UK). Western blots were performed with the specific anti-BrYV movement protein (MP) antibody diluted at 1:1000, and then incubated with goat anti-rabbit IgG (Sigma-Aldrich) diluted at 1:3000. Finally, the membrane was detected by using the NBT and BCIP substrates (Sigma-Aldrich).

\section{Results}

\subsection{Virus inocula were ready for transmission}

There are two BrYV inocula were selected for aphids feeding and transmission. Firstly, the seedlings of the seventh generation transgenic $A$. thaliana line 412 with BrYV full-length cDNA clone grown to 3-4 weeks with all shown typical purple symptoms and were confirmed to carry BrYV by western blotting and RT-PCR which were used as fresh BrYV inocula [14]. And the transgenic plants were also kept at $-20{ }^{\circ} \mathrm{C}$ as frozen BrYV inocula. Secondly, the BrYV full length infectious clone was transferred to Agrobacterium C58CI and was inoculated A. thaliana. After 14 days of inoculation, RTPCR was used to detect BrYV of inoculated leaves and systemic leaves which results were shown that the infection incidence of $A$. thaliana in inoculated leaves by BrYV was $86.34 \%$, and the incidence of systemic leaves was only $46.75 \%$ (Table 1 ). Transgenic $A$. thaliana line 412 and agrobacteriummediated BrYV infectious clone successfully infected $A$. thaliana plants could be used for virus inoculation materials for aphids feeding and transmission.

Table 1. Efficiency of Agro-mediated infiltration of BrYVA in inoculated leaf or system leaves.

\begin{tabular}{cc}
\hline Position & Efficiency (\%) \\
\hline Inoculated leaves $(\mathrm{n}=58)$ & 86.34 \\
Systemic leaves $(\mathrm{n}=58)$ & 46.75 \\
\hline
\end{tabular}

The aphids containing BrYV were identified as green peach aphids (Myzus persicae) and nonviruliferous aphid monoclonal population Mp433-1 was obtained. DNA was extracted from a single aphid and the mitochondrial gene (COI) universal primers (LEP-F and LEP-R) were used for PCR amplification. The sequencing results were identified by blasted on NCBI which was $99 \%$ identity with Myzus persicae strain YL. Cabbage COI (accession number: KM577343) indicating that the aphid specie was green peach aphids (Myzus persicae). Screening the aphid colony (Myzus persicae) was done for separating them to obtain virus-free and monoclonal aphids. One single aphid from the aphid (Myzus persicae) group has been identified species for oviposit and subsequently reared in healthy turnip plant. Then transferred one nymph to healthy $A$. thaliana and for about 5-10 generation, aphids 
were randomly selected and analyzed by RT-PCR for confirming BrYV negatively. For about 5-10 generation offsprings of these non-viruliferous aphids were classified as a monoclonal population named Myzus persicae isolate Mp433-1. The selected aphid offsprings from single aphid were confirmed to be negative by RT-PCR with BrYV specific primers (BrY4964F/ BrY5635R).

\subsection{Acquisition and transmission of $B r Y V$ by the aphids were available}

After feeding aphids with the frozen transgenic A. thaliana line 412 leaves (the preserved time were $180 \mathrm{~d}$ and $270 \mathrm{~d}$ ), fresh line 412 leaves and healthy $A$. thaliana leaves for 2 days (each treatment with 60 aphids). The mean numbers of alive aphids were 35.33, 30.33, 55.33 and 51.33 and with the survival rates of $58.59 \%, 50.56 \%, 92.22 \%$ and $85.56 \%$, respectively (Table 2). Subsequently, sixteen aphids from each treatment was selected randomly in each treatment to inoculate 3-4 weeks-old healthy A. thaliana singly. After 2 days inoculation, the aphids were confirmed by RT-PCR for the presence of BrYV. The mean rates of viruliferous aphids fed on line $412,180 \mathrm{~d}$ and $270 \mathrm{~d}$ frozen infected leaves were $93.75 \%, 87.5 \%, 77.08 \%$, respectively (Table 2). There was no signifiant difference among the above three treatments. The fresh healthy plants were used as negative control. These results showed that both the fresh line 412 and frozen line 412 leaves could be used for feeding aphids for BrYV acquisition, though the frozen leaves affected aphid survival rate compared to the fresh leaves under the same experimental conditions.

Table 2. Percentages of viruliferous aphids and BrYV infected plants transmitted by the aphids that had acquired virus by either feeding on frozen or fresh infected leaves, respectively.

\begin{tabular}{|c|c|c|c|c|}
\hline Treatment & $\begin{array}{l}\text { Total no. of } \\
\text { aphids }\end{array}$ & $\begin{array}{c}\text { No. of } \\
\text { surviving aphids } \\
p<0.001\end{array}$ & $\begin{array}{c}\text { Proportion of } \\
\text { viruliferous aphids } \\
(\mathrm{n}=48)(\%) p=0.0183\end{array}$ & $\begin{array}{l}\text { Proportion of infected } \\
\text { plants } \\
(\mathrm{n}=48)(\%) p=0.0942\end{array}$ \\
\hline Frozen for $180 \mathrm{~d}$ & 180 (60 each) & 106 & $87.5 a$ & $16.6 b$ \\
\hline Frozen for $270 \mathrm{~d}$ & 180 (60 each) & 91 & $77.08 \mathrm{a}$ & $10.4 \mathrm{c}$ \\
\hline $\begin{array}{c}\text { Fresh infected } \\
\text { leaves }\end{array}$ & 180 (60 each) & 166 & $93.75 a$ & 33.33 \\
\hline $\begin{array}{c}\text { Fresh healthy } \\
\text { leaves }\end{array}$ & 180 (60 each) & 154 & - & - \\
\hline
\end{tabular}

Control treatment, total insect number and survivor results are included, BrYV analyzed by RT-PCR. Within a column data followed by the same lowercase letters are significantly different at $p<0.05$.

After 14 days inoculation, sixteen inoculated $A$. thaliana were tested by RT-PCR for BrYV infection. The expected size of the product was amplified from infected plants but not the nosymptom plants (Figure 2a,b). The transmission rates of infected plant by the aphids fed on $412,180 \mathrm{~d}$ and $270 \mathrm{~d}$ frozen infected leaves were $33.33 \%, 16.67 \%, 10.42 \%$, respectively (Table 2). These results indicated that the aphids acquiring the virus from the BrYV genome transgenic plants and frozen infected leaves could transmit the virus to healthy plants, in which the symptoms inoculated by the aphids acquiring BrYV were identical to those of the Agrobacterium-mediated BrYV infection or BrYV genome transgenic plants.

\subsection{The minimal aphid numbers and inoculation time were determined for the best transmission efficiency of $\operatorname{Br} Y V$}

In order to ensure the best transmission efficiency, the transmission efficiency test of inoculation with different numbers of viruliferous aphids was carried out. The aphids were fed to fresh line 412 plants, each of the ten healthy $A$. thaliana plants were inoculated by 1, 2, 4, 6 and 10 aphids (2nd-3rd instar) respectively. After 2 days inoculation, the aphids were eliminated with insecticide. After 14 days inoculation, the results of RT-PCR showed that the rates of infected A. thaliana by $1,2,4,6,10$ aphids were $30 \%, 80 \%, 90 \%, 100 \%$ and $100 \%$, respectively (Table 3 ). The repeated experiments were carried out three times in each group. The results showed that only 6 viruliferous aphids transmitting BrYV for 2 days, which could make the infection efficiency to $100 \%$. Typical symptoms with purple leaves in the vein on $A$. thaliana plants inoculated with 6 viruliferous aphids at 14 dpi (Figure 3). To 
determine the minimal inoculation time of aphid for virus transmission, six viruliferous aphids were tested and inoculated the healthy A. thaliana plants for $6 \mathrm{~h}, 12 \mathrm{~h}, 24 \mathrm{~h}$ and $48 \mathrm{~h}$ respectively. After 14 days inoculation, the RT-PCR results showed that the rates of infected plants by 6 aphids for $6 \mathrm{~h}, 12$ h, $24 \mathrm{~h}$ and $48 \mathrm{~h}$ were $12.5 \%, 50 \%, 62.5 \%$, and $100 \%$ respectively (Table 4 ), indicating that 2 days inoculation six viruliferous aphids could guaranty $100 \%$ infection of BrYV in A. thaliana plants.

Table 3. Virus transmitted efficiency by different numbers of aphids.

\begin{tabular}{ccc}
\hline Total number of insects & No. of Infected plants $(\mathbf{n}=\mathbf{3 0})$ & Proportion of infected plants $(\%)$ \\
\hline 1 & 9 & 30 \\
2 & 24 & 80 \\
4 & 27 & 90 \\
6 & 30 & 100 \\
10 & 30 & 100 \\
\hline
\end{tabular}

a

b

250

251

252

253

254
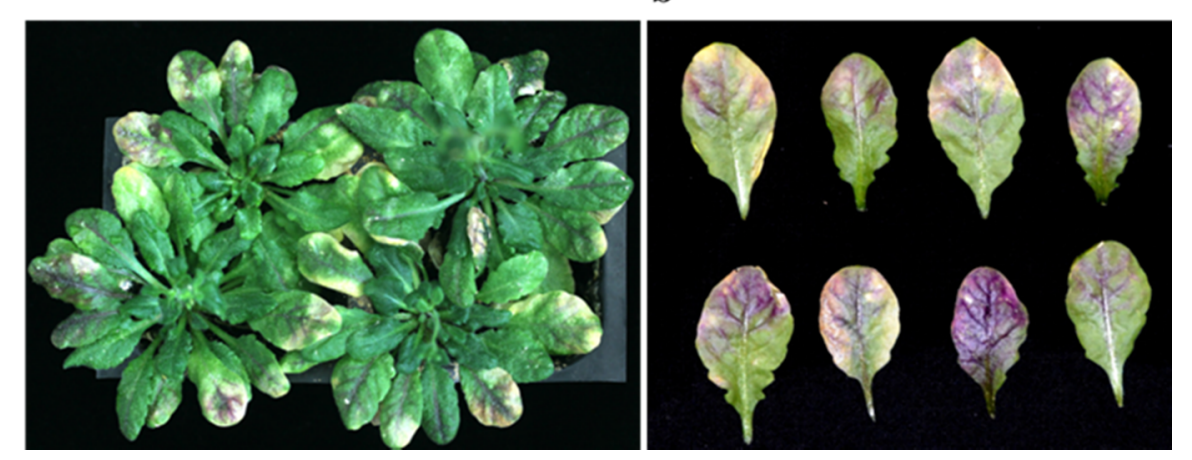

Figure 3. Typical symptoms with purple leaves in the vein on A. thaliana plants inoculated with 6 viruliferous aphids at 14 dpi. (a) The whole plant inoculated with 6 viruliferous aphids; (b) Typical symptoms with purple leaves in the vein.

Table 4. Minimal inoculation time of 6 aphids for virus transmission.

\begin{tabular}{cc}
\hline Inoculation time (h) & Efficiency $(\%)(\mathbf{n}=\mathbf{1 6})$ \\
\hline 6 & 12.5 \\
12 & 50 \\
24 & 62.5 \\
48 & 100 \\
\hline
\end{tabular}

\section{Discussion}

For plant virology research, virus preservation and utilization are very important part $[25,26]$. Plant virus in the Polerovirus (family Luteoviridae) cause emerging diseases with serious economic consequences in many staples, vegetable, ornamental and fruit crops which the transmission by aphids is classified as persistent, circulative, and non-propagative [1,5,6]. For virus acquisition and transmission usually use the virus-infected plants. However, there were not very convenient for tests due to virus inocula preservations and delivers. Therefore, it is important to explore a simple method for the Polerovirus preservation and transmission. The significance of this technique in some ways may easy for virus preserving and transmission by using the virus genomic transgenic model plants and frozen infected leaves. The purpose of preservation is not only to maintain the infectivity of the virus, but to prevent variations of virus from mutation and contamination.

In this research, a very simple, and feasible method was established by using the transgenic $A$. thaliana with BrYV full-length genome and frozen infected leaves for aphid feeding and transmission. 
268 Previous research of Franco-Lara has shown that PLRV viral genomic RNA could transfer into common tobacco and potato which the virus genomic RNA and protein could replication and translation in the transgenic plants indicating that PLRV can be transmitted through seeds. Further, aphids (Myzus persicae) fed on the transgenic tobacco plants readily transmitted PLRV to test plants, and the inoculation efficiency of 5 aphids with 3 days was about 71\% [19]. Boissinot extracted the virus virions from the TuYV (Turnip yellows virus) infected plants to fed aphids through the membrane which used 10 Myzus persicae (Sulzer) and four days for inoculation [17]. For the method is technically demanding and poor operability which should possess virus purification equipment and instruments. In this study, six aphids (Myzus persicae) were used for feeding in BrYV transgenic A. thaliana and two days inoculation which the transmission rate was up to $100 \%$ (Table 3), indicating that transgenic $A$. thaliana plants are more readily to aphids acquiring. More conveniently, BrYV genomic transgenic plants could be germinated from the transgenic $A$. thaliana seeds at any time for aphid-feeding. For virus-infected leaves frozen at $-20^{\circ} \mathrm{C}$ were selected from the BrYV transgenic $A$. thaliana fresh leaves grown to 3-4 weeks or BrYV infected $A$. thaliana, which the preserved time were $180 \mathrm{~d}$ and $270 \mathrm{~d}$. The survival rate of aphids from frozen diseased leaves was $58.89 \%, 50.56 \%$ that was much lower than the aphids fed on the fresh infected leaves and fresh healthy leaves. Though the materials treated under freezing conditions may affect the aphids surviving, there is no significant difference of aphids acquiring virus from the frozen infected leaves (Table 2). The highest survival rate of Myzus persicae in fresh infected leaves may be for that the condition of detached leaves infected by virus could maintain the water loss rate. However, For the transmission rates of the aphids fed on the frozen infected leaves are lower than the fresh infected leaves for about a half indicating that the frozen samples are no picnic for aphids acquiring (Table 2). Zhou's previous work shown that there was no significant difference between the SBPH fed on frozen or fresh infected rice leaves for virus transmission and preserved the infected rice leaves under freezing conditions of $-70{ }^{\circ} \mathrm{C}$ for 45 days and 140 days, which could also be used for SBPH acquiring virus and transmission [22]. Shikata et al (1977) also shown that SBPH could acquire virus from 232 days in frozen leaf tissues of RBSDV infected rice leaves [20]. In this research, a single aphid could acquire the virus from fresh infected leaves and frozen for 270-day infected leaves which was simple and feasible method for aphids acquiring virus at any time and the infected plants could preserved under freezing conditions at -20 ${ }^{\circ} \mathrm{C}$ for at least 270 days. This work maybe be further improved by using aphids to be fed on the leaves of infected by BrYV under $-70{ }^{\circ} \mathrm{C}$ freezing conditions. Agrobacterium tumefaciens-mediated virus inoculation on Arabidopsis thaliana could also be used as virus sources for aphids acquiring BrYV. Since poleroviruses infected plants are very difficult for long-term preservation and utilization, this method overcome the technical bottleneck of virus transmission and preservation which also can be used in screening resistant varieties and genetic analysis of the variety resistance to BrYV. Taken together, this developed method provided a simple and reliable approach by using the BrYV fulllength genome transgenic Arabidopsis and frozen infected leaves for aphid acquiring virus and transmission. This novel method could be not only applied to preservation of virus inocula, evaluation of variety resistance, and biological research on interactions among BrYV, aphid and host, but may provide a new idea on establishing a basic method for other poleroviruses research.

\section{Conclusions}

BrYV can not be transmitted mechanically, in this research, we developed a simple and reliable approach by using the BrYV full-length genome transgenic Arabidopsis thaliana and frozen infected leaves for aphid acquiring virus and transmission. Therefore, this basic research resolves the virus transmission through aphids under natural condition and provides long-term for virus preservation and utilization. This method will also help speed up the research for unraveling interactions among aphid, virus and host and evaluation of variety resistance to virus and aphid.

Author Contributions: H. CG. conceived the study and revised the manuscript. Z. DP. drafted the manuscript, and conducted the experiments, C. XR., H. RJ., Z. TY., Z. XY, P. YM. provided partial experiment materials. W. Y., L. DW., and Y. JL. analyzed the data. All authors read and approved the final manuscript. 
Funding: This work was supported by the National Natural Science Foundation of China (31671995 and 31972240).

Acknowledgments: We are grateful to Professor Rao (University of California, U.S.A.) for providing the vector pCass4-Rz. Xiao-Rong Tao (Nanjing Agricultural University, China) for providing pCB301-2x35S-MCS-HDVRzNOS vector; Dao-Wen Wang (China Academy of Agricultural Science, China) for providing C58CI. We also thank Savithramma P. Dinesh-Kumar (University of California, Davis, U.S.A.), Shou-Wei Ding (University of California, Riverside, U.S.A.) for their helpful comments on this research. This work was supported in part by the National Natural Science Foundation of China (31671995 and 31972240).

Conflicts of Interest: The authors declare no conflict of interest.

\section{References}

1. Heck, M.; Brault, V. Targeted disruption of aphid transmission: a vision for the management of crop diseases caused by Luteoviridae members. Curr Opin Virol, 2018, 33, 24-32.

2. D'Arcy, C.J.; Domier, L.L. Family Luteoviridae. In Virus Taxonomy: Eighth Report of the International Committee on Taxonomy of Viruses; Fauquet, C.M., Mayo, M.A., Maniloff, J., Desselberger, U., Ball, L.A., Eds.; Academic Press: Waltham, MA, USA, 2005; pp. 343-352.

3. Mauck, K. E., Variation in virus effects on host plant phenotypes and insect vector behavior: what can it teach us about virus evolution? Curr Opin Virol, 2016, 21, 114-123.

4. Yoshida, N.; Tamada, T. Host range and molecular analysis of Beet leaf yellowing virus, Beet western yellows virus-JP and Brassica yellows virus in Japan. Plant Pathol, 2019, 68, 1045-1058.

5. Taliansky, M.; Mayo, M.; Barker, H. Potato leafroll virus: a classic pathogen shows some new tricks. Mol Plant Pathol. 2003, 4(2), 81-89.

6. Mayo, M.; Ryabov, E.; Fraser, G.; Taliansky, M. Mechanical transmission of Potato leafroll virus. J. Gen. Virol. 2000, 81, 2791-2795.

7. Gray, S.; Gildow, F. E., Luteovirus-aphid interactions. Annu Rev Phytopathol, 2003, 41, 539-66.

8. Xiang, H.Y.; Dong, S.W.; Shang, Q.X.; Zhou, C.J.; Li, D.W., Yu, J.L., Han, C.G. Molecular characterization of two genotypes of a new polerovirus infecting brassicas in China. Arch. Virol. 2011, 156, 2251-2255.

9. Wang, Q.; Mao, J.; Xiang, H.; Dong, L.H.; Sun, Y.H.; Liu, G.S.; Liu, H.B. First report of Brassica yellows virus on tobacco in China. Plant Dis, 2015, 99, 1192.

10. Zhang, X.; Peng, Y.; Wang, Y.; Zhang, Z.; Li, D.; Yu, J.; Han, C. Simultaneous detection and differentiation of three genotypes of Brassica yellows virus by multiplex reverse transcription-polymerase chain reaction. Virol J, 2016, 13 (1), 189.

11. Lim, S.; Yoo, R. H.; Igori, D.; Zhao, F.; Kim, K. H.; Moon, J. S. Genome sequence of a recombinant brassica yellows virus infecting Chinese cabbage. Arch. Virol, 2015, 160, 597-600.

12. Zhang X.Y.; Xiang, H.Y.; Zhou, C.J.; Li, D.W.; Yu, J.L.; Han, C.G. Complete genome sequence analysis identifies a new genotype of brassica yellows virus that infects cabbage and radish in China. Arch. Virol, 2014, 159, 2177-2180.

13. Zhang, X.Y.; Dong, S.W.; Xiang, H.Y.; Chen, X.R.; Li D.W.; Yu, J.L.; Han, C.G. Development of three fulllength infectious cDNA clones of distinct brassica yellows virus genotypes for agrobacterium-mediated inoculation. Virus Res, 2015, 197, 13-16.

14. Chen, X. R.; Wang, Y.; Zhao, H. H.; Zhang, X.Y.; Wang, X.B.; Li, D.W.; Yu, J.L.; Han, C.G. Brassica yellows virus' movement protein upregulates anthocyanin accumulation, leading to the development of purple leaf symptoms on Arabidopsis thaliana. Sci Rep, 2019, 8:16273.

15. Li, Y.Y.; Sun, Q.; Zhao, T.Y.; Xiang, H.Y.; Zhang X.Y.; Wu, Z.Y.; Zhou, C.J.; Zhang, X., Wang, Y.; Zhang Y.L.; Wang, X.B.; Li D.W.; Yu, J.J.; Dinesh-Kumar, S.P.; Han, C.G. Interaction between Brassica yellows virus silencing suppressor $\mathrm{P} 0$ and plant SKP1 facilitates stability of P0 in vivo against degradation by proteasome and autophagy pathways. New Phytol, 2019, 222 (3):1458-1473.

16. Sun, Q.; Li, Y.Y.; Wang, Y.; Zhao, H.H.; Zhao, T.Y.; Zhang, Z.Y.; Li, D.W.; Yu, J.L.; Wang, X.B.; Zhang, Y.L.; Han, C.G. Brassica yellows virus P0 protein impairs the antiviral activity of NbRAF2 in Nicotiana benthamiana. J EXP BOT, 2019, 69 (12): 3127-3139.

17. Boissinot, S.; Pichon, E.; Sorin, C.; Piccini, Céline.; Scheidecker, D.; Ziegler-Graff, V.; Brault, V. Systemic Propagation of a Fluorescent Infectious Clone of a Polerovirus Following Inoculation by Agrobacteria and Aphids. Viruses, 2017, 9, 166. 
18. Van den Heuvel, J.F.J.M.; Boerma, T.M.; Peters, D. Transmission of potato leafroll virus from plants and artificial diets by Myzus persicae. Phytopathology, 1991, 81, 150-154.

19. Franco-Lara, L.; D.McGeachy, K.; Commandeur, U.; Martin, R.R.; Mayo,M.A.; Barker, H. Transformation of tobacco and potato with cDNA encoding the full-length genome of Potato leafroll virus: evidence for a novel virus distribution and host effects on virus multiplication. J. Gen. Virol, 1999, 80, 2813-2822.

20. Shikata, E.; Kitagawa, Y. Rice black-streaked dwarf virus: Its properties, morphology and intracellular localization. Virology, 1977, 77(2):826-842.

21. Zhang, S.X.; Li, L.; Wang, X.F.; Zhou, G.H. Transmission of Rice stripe virus acquired from frozen infected leaves by the small brown planthopper (Laodelphax striatellus Fallen). J VIROL METHODS, 2007, 146: 359362.

22. Zhou, T.; Wu, L.J.; Wang, Y.; Cheng, Z.B.; Ji, Y.H.; Fan, Y.J.; Zhou, Y.J. Transmission of rice black streaked dwarf virus from frozen infected leaves to healthy rice plants by small brown Planthopper (Laodelphax striatellus). Rice Science, 2011,18(2): 152-156.

23. Srinivasan, R.; Alvarez, J. M.; Bosque-Pérez, N. A.; Eigenbrode, S. D.; Novy, R. G. Effect of an Alternate Weed Host, Hairy Nightshade, Solanum sarrachoides (Sendtner), on the Biology of the Two Most Important Potato Leafroll Virus (Luteoviridae: Polerovirus) Vectors, Myzus persicae and Macrosiphum euphorbiae (Aphididae: Homoptera). Environ Entomol, 2008, 37 (2), 592-600.

24. Van den Heuvel, J.F.J.M.; Boerma, T.M.; Peters, D. Transmission of potato leafroll virus from plants and artificial diets by Myzus persicae. Phytopathology 1991, 81, 150-154.

25. Hollings M.; Stone, M. The long-term survival of some plant viruses preserved by lyophilization. Ann Appl Boil, 1970, 65:411-418.

26. Hollings M.; Lelliott, R.A. Preservation of plant viruses by freeze drying. Pl Path, 1960, 9:63-66. 